\title{
Video Article \\ Fluorescence Recovery After Photobleaching (FRAP) of Fluorescence Tagged Proteins in Dendritic Spines of Cultured Hippocampal Neurons
}

\author{
Chan-Ying Zheng ${ }^{1}$, Ronald S. Petralia ${ }^{1}$, Ya-Xian Wang ${ }^{1}$, Bechara Kachar ${ }^{1}$ \\ ${ }^{1}$ National Institute on Deafness and Other Communication Disorders, National Institutes of Health, Bethesda \\ Correspondence to: Chan-Ying Zheng at zhengchan@mail.nih.gov \\ URL: https://www.jove.com/video/2568 \\ DOI: doi:10.3791/2568
}

Keywords: Neuroscience, Issue 50, Spine, FRAP, hippocampal neurons, live cell imaging, protein mobility

Date Published: 4/16/2011

Citation: Zheng, C.Y., Petralia, R.S., Wang, Y.X., Kachar, B. Fluorescence Recovery After Photobleaching (FRAP) of Fluorescence Tagged Proteins in Dendritic Spines of Cultured Hippocampal Neurons. J. Vis. Exp. (50), e2568, doi:10.3791/2568 (2011).

\section{Abstract}

FRAP has been used to quantify the mobility of GFP-tagged proteins. Using a strong excitation laser, the fluorescence of a GFP-tagged protein is bleached in the region of interest. The fluorescence of the region recovers when the unbleached GFP-tagged protein from outside of the region diffuses into the region of interest. The mobility of the protein is then analyzed by measuring the fluorescence recovery rate. This technique could be used to characterize protein mobility and turnover rate.

In this study, we express the (enhanced green fluorescent protein) EGFP vector in cultured hippocampal neurons. Using the Zeiss 710 confocal microscope, we photobleach the fluorescence signal of the GFP protein in a single spine, and then take time lapse images to record the fluorescence recovery after photobleaching. Finally, we estimate the percentage of mobile and immobile fractions of the GFP in spines, by analyzing the imaging data using ImageJ and Graphpad softwares.

This FRAP protocol shows how to perform a basic FRAP experiment as well as how to analyze the data.

\section{Video Link}

The video component of this article can be found at https://www.jove.com/video/2568/

\section{Neuron transfection}

1. Culture embryonic day 18 (E18) rat hippocampal neurons on poly-d-lysine-coated MatTek 35 -mm glass-bottom dishes ${ }^{1}$. On $16-18$ days in vitro(DIV), transfect neurons using the Clontech CalPhos Mammalian Transfection Kit. First, replace the culture medium with $1.5 \mathrm{ml}$ Dulbecco's Modified Eagle Medium (DMEM) per 35-mm dish 0.5 hour prior to transfection. Save the original culture medium in a sterile $15 \mathrm{ml}$ tube for later (step 1.6) use.

2. Mix $10 \mu \mathrm{g}$ pEGFP-N1 plasmid DNA with sterile $\mathrm{H}_{2} \mathrm{O}$ (Clontech) and $12.4 \mu \mathrm{l} 2 \mathrm{M}$ calcium solution (Clontech) to a total volume of $100 \mu \mathrm{\mu l}$.

3. Add the mixture from step 1.2) to $100 \mu \mathrm{l} 2 \times \mathrm{HBS}$ dropwise while vortexing $2 \times \mathrm{HBS}$ at medium speed.

4. Let the mixture sit at room temperature for 20 minutes and then add the final mixture from step 1.3) into DMEM-incubated neurons.

5. Put the neurons back into the $37^{\circ} \mathrm{C}$ incubator for $1-1.5$ hours.

6. Remove the calcium phosphate-containing medium, then wash cells with DMEM three times. Before returning the culture dish to the incubator, exchange the DMEM medium with the original culture medium.

\section{FRAP experiment on a spine}

1. Neurons are used for the FRAP experiment two to four days after transfection.

2. Replace the culture medium from the $35-\mathrm{mm}$ glass-bottom dish, by immediately adding pre-warmed Tyrode Solution, which contains (in mM) $\mathrm{NaCl} 145, \mathrm{KCl} 5$, HEPES 10, Glucose 10, Glycine 0.005, $\mathrm{CaCl}_{2} 2.6$, and $\mathrm{MgCl}_{2} 1.3$ (pH adjusted to 7.4 with $\mathrm{NaOH}$ ).

3. A Zeiss LSM 710 confocal microscope is used for the FRAP experiment. The Zeiss TempModule system is used to control the temperature $\left(37^{\circ} \mathrm{C}\right)$, the humidity and the $\mathrm{CO}_{2}(5 \%)$ of the working system. Make sure that the $\mathrm{CO}_{2}$ tank is connected and the water bottle, which is used for balancing humidity, is filled with water.

4. Find a transfected mature dendrite with the $100 \times$ objective (aplan-APOCHROMAT $100 \times / 1.46$ oil). If the transfected cells in the dish are sparse, search for a desired cell with the $40 \times$ objective (plan-NEOFLUAR $40 \times / 1.3$ oil), and then switch to the $100 \times$ objective to capture images.

5. Use $5 \times$ optical zoom and a $256 \times 256$ pixel resolution to image a short piece of dendrite with several spines. To capture images, use nominal speed 9 (pixel dwell time $3.15 \mu \mathrm{sec}$ ) which takes 0.5 second to finish a scan. The pinhole is set to $2 \mu \mathrm{m}$ to obtain strong fluorescence. When taking images, try to use low laser transmission, for example 1-5\%, to avoid photobleaching the entire image. 
6. Select the spine of interest. In our experiment, we choose mushroom spines with their spine head diameters of $\sim 1 \mu \mathrm{m}$.

7. To do a FRAP experiment, take 5 control images before bleaching, then bleach the spine of interest 10 times at nominal $100 \%$ laser transmission [The argon laser power is $30 \mathrm{~mW}$, with $50 \%(15 \mathrm{~mW})$ in the 488 laser line, and approximately $3-4 \mathrm{~mW}$ reaches the microscope through the 488 laser line], and then capture a series of images immediately after bleaching. For this experiment, images are captured every 1 second for 15 seconds after bleaching. The interval of time should be adjusted according to different targeting proteins and different experimental designs (Fig. 1).

8. Save images.

\section{Data analysis}

1. Open images with ImageJ software.

2. Align the stack of images using the align tool ('plugins' $\rightarrow$ 'stacks - shuffling' $\rightarrow$ 'align slices in stack' $\rightarrow$ 'translation' and/or 'rigid body') in ImageJ, so that the spine of interest does not float, in other words - it remains in the same position on the image.

3. Measure relative fluorescence intensity of the spine of interest $\left(F_{s}\right)$, a transfected but unbleached region (control), and a background region $\left(F_{b}\right)$ in time lapse images, using the 'Intensity $\vee$ Time Monitor' tool of ImageJ ('plugins' $\rightarrow$ 'stacks - shuffling' $\rightarrow$ 'Intensity $\vee$ Time Monitor'). The control region could be a piece of well-focused distal dendrite. The background region is a non-fluorescent region.

4. Calculate the photobleaching rate $(r)$ by comparing the fluorescence of the control region before $\left(F_{c 0}\right)$ and after $\left(F_{c}\right)$ photobleaching $r=F_{c} / F_{c 0}$.

5. Normalize the fluorescence intensity of the target spine $(F)$ as follows: $F=\left(F_{s}-F_{b}\right) / r$.

6. Curve fit the fluorescence intensity of the target spine with a one-phase exponential equation of Graphpad Prism software (Fig. 2) or other similar software.

7. Calculate the mobile fraction $\left(f_{m}\right)$ and the immobile fraction $\left(f_{i}\right)$ by the following equations: $f_{m}=F_{\infty} / F_{0}$, where $F_{\infty}$ is the fluorescence intensity after full recovery, and $F_{0}$ is the fluorescence intensity before photobleaching. $f_{i}=1-f_{m}$

\section{Representative Results:}

In this study, we perform a FRAP experiment on mature hippocampal neurons. At 18-22 DIV, mushroom spines are already formed. Using our method, the dynamic changes of the fluorescence intensity in a small region, such as a spine, can be recorded.

To analyze the fluorescence recovery process of EGFP, we take 5 images as controls before bleaching and then 1 image every 1 second immediately after bleaching for 15 seconds. The resolution of the image is sufficent for quantitative analysis. The fluorescence recovery profiles of tagged fluorescence proteins are highly reproducible.

We also briefly show how to define the mobile and immobile fractions of a fluorescence protein, using ImageJ and Graphpad Prism software. The FRAP method and analysis we show here can be broadly used in neuroscience, cell biology and other studies.
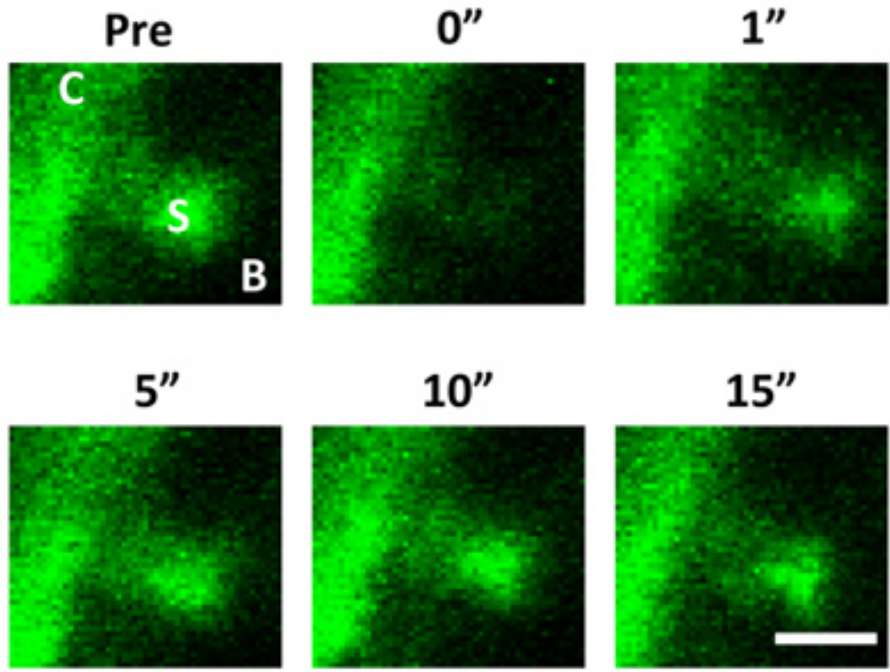

Figure 1. FRAP measurements of EGFP fluorescence in a spine from a cultured hippocampal neuron. The red arrowheads indicate the time of photobleaching. Photographs represent the same area before (Pre) and at 0,1,5, 10, 15 seconds after photobleaching. The region of the spine, control and background are marked with letters S, C and B, respectively. Neurons were maintained at $37^{\circ} \mathrm{C}$ during the experiment. Scale bar, 1 $\mu \mathrm{m}$. 


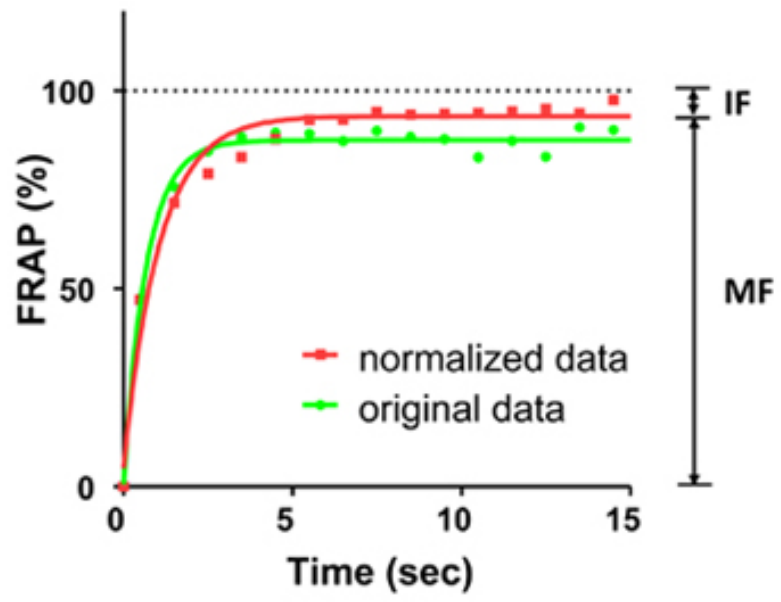

Figure 2. FRAP curves of EGFP fluorescence over a 15-second period. The green line shows the original curve; the red line shows the normalized curve. The dots on curves show the FRAP every 1 second. The curves were fitted by one-phase exponential equations. The average fluorescence before photobleaching was counted as $100 \%$. In this experiment, the mobile fraction (MF) is $94 \%$ and the immobile fraction (IF) is $6 \%$.

\section{Discussion}

FRAP analysis has been broadly used in vivo and in vitro ${ }^{1-2}$ studies. This technique commonly utilizes GFP fusion proteins, although it could also use red alga fusion proteins ${ }^{3}$. This analysis is sensitive and can be used to characterize the mobility of GFP-tagged proteins.

To produce meaningful FRAP analysis, it is important to avoid unnecessary photobleaching before and during the FRAP experiment. There are two ways to achieve this. First, the process to search and observe the experimental neuron should be fast. Especially, observation of neurons with a 100X objective for a long time significantly bleaches the fluorescence. Second, high laser power and frequent scanning often increase the possibility of photobleaching. Thus, it will be necessary to calculate the photobleaching rate in a control region and then normalize the FRAP curve in the experimental region. The normalizing method has been described in the protocol (see step 3.3-3.5 for details).

The photobleaching step is also critical for ensuring good FRAP results. In this experiment, we bleach the spine of interest 10 times at $100 \%$ laser transmission. This condition is sufficient to bleach the fluorescence of a spine to background level in a fixed preparation. Thus, we set the fluorescence intensity to the same number as background at 0 seconds after photobleaching. Depending on the speed of the first scan, a significant amount of fluorescence recovery might already be detected when the protein of interest is highly mobile.

Many fluorescent protein probes have been developed to study protein dynamics with FRAP. A complementary approach is, for example, to use photoactivatable GFP (PA-GFP), or photoconvertible variants. Together with FRAP technique, these tools are becoming indispensible for live cell imaging studies ${ }^{4-6}$.

\section{Disclosures}

No conflicts of interest declared.

\section{Acknowledgements}

This work was supported by the National Institute on Deafness and Other Communication Disorders (NIDCD) Intramural Program.

\section{References}

1. Zheng, C. Y., Petralia, R. S., Wang, Y. X., Kachar, B. \& Wenthold, R. J. SAP102 is a highly mobile MAGUK in spines. J Neurosci $\mathbf{3 0}$, 4757-4766, (2010).

2. Grati, M. et al. Rapid turnover of stereocilia membrane proteins: evidence from the trafficking and mobility of plasma membrane Ca(2+)ATPase 2. J Neurosci 26, 6386-6395, (2006).

3. Liu, L. N., Aartsma, T. J., Thomas, J. C., Zhou, B. C., \& Zhang, Y. Z. FRAP Analysis on Red Alga Reveals the Fluorescence Recovery Is Ascribed to Intrinsic Photoprocesses of Phycobilisomes than Large-Scale Diffusion. PLoS ONE 4, e5295, (2009).

4. Wiedenmann, J. \& Nienhaus, G. U. Live-cell imaging with EosFP and other photoactivatable marker proteins of the GFP family. Expert Rev Proteomics 3, 361-374, (2006).

5. Shaner, N. C., Patterson, G. H. \& Davidson, M. W. Advances in fluorescent protein technology. J Cell Sci 120, 4247-4260, (2007).

6. Sprague, B. L. \& McNally, J. G. FRAP analysis of binding: proper and fitting. Trends Cell Biol 15, 84-91, (2005). 